\title{
Sliding Mode Control with a State Predictor for Time Delay Control System
}

\author{
T. Ishibashi, K. Kawaguchi, H. Shibasaki, R. Tanaka, T. Murakami, and Y. Ishida
}

\begin{abstract}
In this study, we propose a Sliding Mode Control (SMC) with a state predictor for time delay control systems. Time delay is a factor that causes instability in systems, and SMC is weakened by time delay. Therefore, we use a state predictor with SMC. State predictors are easily designed, and are suitable in combination with SMCs using the state of the plant as a controller. We have successfully realized control using a SMC with a state predictor for the plant with the time delay. In addition, we have confirmed the robustness of the proposed method. The proposed method shows the effective result compared with the conventional method in the simulation.
\end{abstract}

Index Terms-Time delay, sliding mode control, State predictor.

\section{INTRODUCTION}

The theory of a Sliding Mode Control (SMC) is based on the concept of changing the structure of a controller in response to the changing state of a system with the aim of obtaining a desired response. A high-speed switching control action is used to switch between different structures, and the trajectory of the system is forced to move along a chosen manifold in the state space, called the switching surface. Therefore, the behavior of the closed loop system is determined by the sliding surface [1]-[3]. In addition, SMC is a nonlinear robust control that attempts to stabilize the system by limiting the state to a switching hyperplane. One of its features is excellent robustness to disturbances and the uncertainties that fulfill the matching condition.

However, according to the principle of SMC, the oscillatory phenomenon called chattering is produced because the existence of a time delay might influence the high-speed switch of the control input, which limits the state to the switching hyperplane [4].

When SMC is applied to an actual system, the state of the system causes oscillations such as high-frequency vibrations and spill-over near the switching hyperplane when chattering occurs, and might lead to the deterioration of the controller.

In this study, we design a state predictor and use SMC to determine the predicted states of the system. A state predictor can be easily designed because we only have to add a predictor to the conventional observer.

This paper is organized as follows: In section II, we describe the design of the system in this study. In section III, the servo system is designed, allowing the state to follow a reference signal. In section IV, we describe the design of the

Manuscript received November 21, 2012; revised January 23, 2013.

T. Ishibashi is with School of Science and Technology, Meiji University, Kawasaki-shi, Kanagawa, 214-8571 Japan (e-mail: ee91053@meiji.ac.jp).
SMC. In section $\mathrm{V}$, we explain the state predictor. In section VI, we present the entire design as a proposed method. In section VII, we compare the simulation results of the proposed method and conventional method, and we confirm the robustness of SMC with the state predictor. Finally, section VIII concludes the paper.

\section{State Space Representation of Controlled SYSTEM}

In this section, the transfer function of the plant $G(s)$ is

$$
\begin{aligned}
G(s) & =\frac{b}{s(s+a)} e^{-L s} \\
& =G_{p}(s) e^{-L s} .
\end{aligned}
$$

$L \in \mathbb{R}^{1 \times 1}$ is the time delay and $a, b \in \mathbb{R}^{1 \times 1}$ is the plant parameter. $e \in \mathbb{R}^{1 \times 1}$ is an exponential function, and $G_{p}(s)$ is the system not including the time delay. The transfer function is rewritten in state space representation for the designing of the controller as

$$
\left\{\begin{array}{c}
\dot{\boldsymbol{x}}(t)=\boldsymbol{A x}(t)+\boldsymbol{B} u(t-L) \\
y(t)=\boldsymbol{C} \boldsymbol{x}(t)
\end{array}\right.
$$

where

$$
\begin{aligned}
& \boldsymbol{A} \in \mathbb{R}^{2 \times 2}, \quad \boldsymbol{B} \in \mathbb{R}^{2 \times 1}, \quad \boldsymbol{C} \in \mathbb{R}^{1 \times 2}, \\
& \boldsymbol{x}(t)=\left[\begin{array}{l}
x_{1}(t) \\
x_{2}(t)
\end{array}\right] \in \mathbb{R}^{2 \times 1}, \text { and } \quad \boldsymbol{x}(0)=\boldsymbol{0} .
\end{aligned}
$$

$y(t) \in \mathbb{R}^{1 \times 1}$ is an output from the plant. In this section, the time delay exists at the input side of the plant, and the plant is a single-input single-output (SISO) system. $\boldsymbol{x}(t)$ is a state vector, and $u(t) \in \mathbb{R}^{1 \times 1}$ is an input variable.

\section{Design OF SERVo System}

A state space is designed by a spreading system as

$$
\left\{\begin{array}{c}
\dot{\boldsymbol{x}}_{e}(t)=\boldsymbol{A}_{e} \boldsymbol{x}_{e}(t)+\boldsymbol{B}_{e} u(t-L) \\
y_{e}(t)=\boldsymbol{C}_{e} \boldsymbol{x}_{e}(t)
\end{array}\right.
$$

where

$$
\begin{aligned}
& \boldsymbol{A}_{e}=\left[\begin{array}{cc}
\boldsymbol{A} & 0 \\
-\boldsymbol{C} & 0
\end{array}\right] \in \mathbb{R}^{3 \times 3}, \quad \boldsymbol{B}_{e}=\left[\begin{array}{c}
\boldsymbol{B} \\
0
\end{array}\right] \in \mathbb{R}^{3 \times 1}, \\
& \boldsymbol{C}_{e}=\left[\begin{array}{ll}
0 & 1
\end{array}\right] \in \mathbb{R}^{1 \times 3}, \quad \text { and } \quad \boldsymbol{x}_{e}(t)=\left[\begin{array}{c}
\boldsymbol{x}(t) \\
z(t)
\end{array}\right] \in \mathbb{R}^{3 \times 1} ;
\end{aligned}
$$

let

$$
z(t)=\int(r(t)-y(t)) d t
$$




$$
\dot{z}(t)=r(t)-y(t)
$$

$r(t) \in \mathbb{R}^{1 \times 1}$ is a reference variable. (3.1) is called the servosystem.

The feedback gain $\boldsymbol{F}_{1}$ and servo gain $F_{2}$ represent the relative importance of the state variation and control energy consumption, respectively, and are given by the optimal control method as

$$
J=\int_{0}^{\infty}\left\{\boldsymbol{x}_{e}(t)^{T} \boldsymbol{Q}_{e} \boldsymbol{x}_{e}(t)+r_{e} u(t)^{2}\right\} d t
$$

where $\boldsymbol{Q}_{e} \in \mathbb{R}^{3 \times 3}$ is a positive definite symmetric matrix, and $r_{e} \geq 0$. If the optimal state feedback gain $\boldsymbol{F}_{\boldsymbol{1}}$ and servo gain $F_{2}$, which minimize the performance index $J$ in (3.2), are rewritten as $\boldsymbol{F}=\left[\begin{array}{ll}\boldsymbol{F}_{1} & F_{2}\end{array}\right], \boldsymbol{F}$ is given by

$$
\boldsymbol{F}=-r_{e}^{-1} \boldsymbol{B}_{e}^{T} \boldsymbol{P}_{e}
$$

where $\boldsymbol{F} \in \mathbb{R}^{1 \times 3}, \boldsymbol{F}_{1} \in \mathbb{R}^{1 \times 2}$, and $F_{2} \in \mathbb{R}^{1 \times 1}$.

The matrix $\boldsymbol{P}_{e}$ is the solution of the revised Riccati equation, and is given as

$$
\boldsymbol{P}_{e} \boldsymbol{A}_{e}+\boldsymbol{A}_{e}^{T} \boldsymbol{P}_{e}-\boldsymbol{P}_{e} \boldsymbol{B}_{e} r_{e}^{-1} \boldsymbol{B}_{e}^{T} \boldsymbol{P}_{e}+\boldsymbol{Q}_{e}=\mathbf{0}
$$

where $\boldsymbol{P}_{e} \in \mathbb{R}^{3 \times 3}[5]-[8]$.

\section{SLIDING Mode CONTROL}

Sliding mode control is expressed as

$$
\left\{\begin{array}{c}
\dot{\boldsymbol{x}}(t)=\boldsymbol{A x}(t)+\boldsymbol{B} u(t) \\
\sigma(t)=\boldsymbol{S} \boldsymbol{x}(t)
\end{array}\right.
$$

$\sigma(t) \in \mathbb{R}^{1 \times 1}$ is the switching surface and $S \in \mathbb{R}^{1 \times 2}$ is the switching matrix.

\section{A. Design of Switching Matrix}

First, to realize control using a sliding mode controller, we are required to design a switching matrix $\boldsymbol{S} . \boldsymbol{S}$ is determined by solving the Riccati equation by employing a method that uses the system's zero- point. The Riccati equation is given as

$$
\boldsymbol{P}_{S} \boldsymbol{A}_{*}+\boldsymbol{A}_{*}^{T} \boldsymbol{P}_{S}-\boldsymbol{P}_{S} \boldsymbol{B} r_{S}^{-1} \boldsymbol{B}^{T} \boldsymbol{P}_{S}+\boldsymbol{Q}_{S}=\mathbf{0}
$$

where

$$
\begin{gathered}
\boldsymbol{A}_{*}=\boldsymbol{A}+\varepsilon \boldsymbol{I}, \varepsilon \in \mathbb{R}^{1 \times 1} \geq 0 \\
\boldsymbol{S}=\boldsymbol{B}^{T} \boldsymbol{P}_{S}
\end{gathered}
$$

Let $\boldsymbol{P}_{S} \in \mathbb{R}^{2 \times 2}, \boldsymbol{I}=\left[\begin{array}{ll}1 & 0 \\ 0 & 1\end{array}\right] \in \mathbb{R}^{2 \times 2}$, and $r_{s} \in \mathbb{R}^{1 \times 1}$.

Using this method, $\boldsymbol{S}\left(\boldsymbol{I} \boldsymbol{I}-\boldsymbol{A}^{T}+\boldsymbol{B S}\right)^{-1} \boldsymbol{B}$ becomes strict positive realness, so that its zero is stable [6].

\section{B. Design of Sliding Mode Controller}

The control input $u(\mathrm{t})$ consists of a linear input $u_{l}(t) \in \mathbb{R}^{1 \times 1}$ and nonlinear- input $u_{n l}(t) \in \mathbb{R}^{1 \times 1}$. That is,

$$
u(t)=u_{l}(t)+u_{n l}(t)
$$

$u_{l}(t)$ is designed as a control system of equal value, i.e.,

$$
u_{l}(t)=-(\boldsymbol{S B})^{-1} \boldsymbol{S} \boldsymbol{A} \boldsymbol{x}(t)
$$

$u_{n l}(t)$ is designed as a nonlinear control input as

$$
u_{n l}(t)=-K \frac{\sigma(t)}{|\sigma(t)|}
$$

$K \in \mathbb{R}^{1 \times 1}$ is a gain of the nonlinear input.

\section{Prevention of the Chattering Phenomenon}

If a nonlinear control input is designed using (4.3b), a chattering phenomenon occurs. When $\sigma(t)=0$ for $u_{n l}(t)$, $|\sigma(t)|$ in the denominator becomes 0 , and quick switching of the input occurs. This phenomenon is called chattering, which is a high-frequency vibrations. To avoid this problem, (4.3b) is written as

$$
u_{n l}(t)=-K \frac{\boldsymbol{\sigma}(t)}{|\boldsymbol{\sigma}(t)|+\boldsymbol{\eta}}
$$

where $\eta \in \mathbb{R}^{1 \times 1}, \eta>0$. (4.1c) becomes a smooth function and the chattering phenomenon can be relieved [9]-[14].

\section{State Predictor}

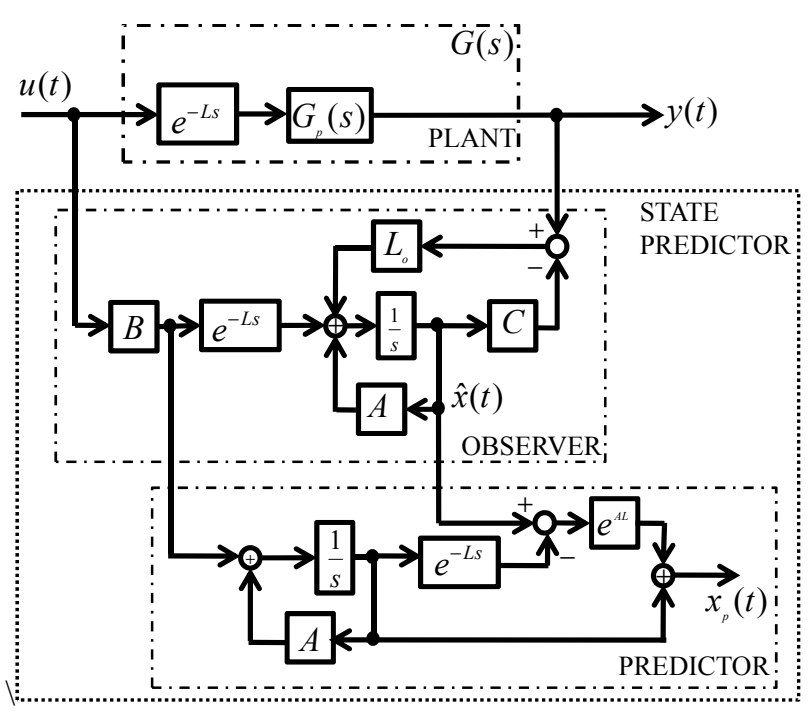

Fig. 1. Block diagram of the state predictor.

Fig. 1 shows the block diagram of the state predictor. Pole placements and optimal control problems for the system with the delay time at the input and output, can be easily settled by using predictive states. A state predictor is designed as

$$
\begin{array}{r}
\dot{\hat{\boldsymbol{x}}}(t)=\boldsymbol{A} \hat{\boldsymbol{x}}(t)+\boldsymbol{B} u(t-L)+\boldsymbol{L}_{o}(y(t)-\hat{y}(t)) \\
\hat{y}(t)=\boldsymbol{C} \hat{\boldsymbol{x}}(t) \\
\dot{\boldsymbol{x}}_{p}(t)=\boldsymbol{A} \boldsymbol{x}_{p}(t)+\boldsymbol{B} u(t)+\boldsymbol{L}_{p}(y(t)-\hat{y}(t))
\end{array}
$$

where $\hat{\boldsymbol{x}}(\mathrm{t}) \in \mathbb{R}^{2 \times 1}$ is the observer state and $\boldsymbol{x}_{p}(t) \in \mathbb{R}^{2 \times 1}$ is the predicted state. $\boldsymbol{L}_{o}, \boldsymbol{L}_{p} \in \mathbb{R}^{2 \times 1}$ are predictor gains and are satisfied with 


$$
\boldsymbol{L}_{p}=\boldsymbol{\Phi}(L) \boldsymbol{L}_{o}
$$

$\boldsymbol{\Phi}(t) \in \mathbb{R}^{2 \times 2}$ is a transition matrix of (5.1). i.e.,

$$
\boldsymbol{\Phi}(t)=e^{A t}
$$

$\boldsymbol{L}_{o}$ is required to be the same the observer gain. i.e.,

$$
\boldsymbol{L}_{o}^{T}=\left(\boldsymbol{C P}_{o} \boldsymbol{C}^{T}+r_{o}^{-1}\right) \boldsymbol{C} \boldsymbol{P}_{o} \boldsymbol{A}
$$

$\boldsymbol{P}_{o} \in \mathbb{R}^{2 \times 2}$ is given by Riccati equation as

$$
\boldsymbol{P}_{o} \boldsymbol{A}+\boldsymbol{A}^{T} \boldsymbol{P}_{o}-\boldsymbol{P}_{o} \boldsymbol{B}_{o}^{-1} \boldsymbol{B}^{T} \boldsymbol{P}_{o}+\boldsymbol{Q}_{o}=\mathbf{0}
$$

where $\boldsymbol{Q}_{o} \in \mathbb{R}^{2 \times 2}, r_{o} \in \mathbb{R}^{1 \times 1}$.

(5.1) represents an ordinary observer, and (5.3) represents a predictor that is driven by the state observation error. Together with (5.2) and (5.3), it is called a state predictor [15].

\section{PROPOSED METHOD}

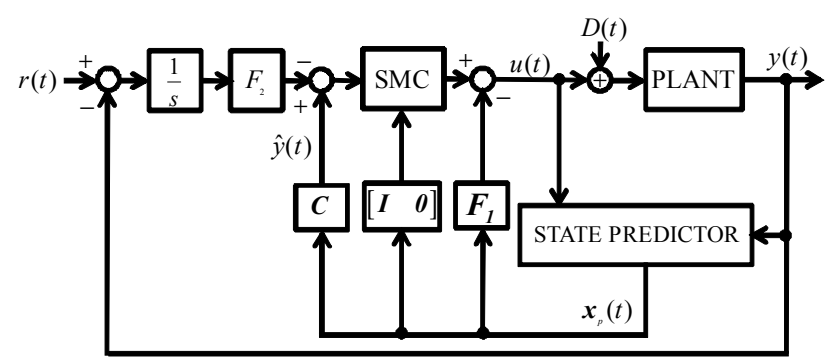

Fig. 2. Block diagram of the proposed method.

Fig. 2 shows the block diagram of the proposed method. $D(t) \in \mathbb{R}^{1 \times 1}$ is an input side disturbance. We use a vector $\left[\begin{array}{ll}\text { I } & 0\end{array}\right] \in \mathbb{R}^{1 \times 2}$ because SMC requires states of the plant model; however, output of the plant model is not required. The output is required by servo system.

\section{Simulation Results}

In this study, the transfer function is defined as

$$
G(s)=\frac{339.6}{s(s+10.78)} e^{-0.04}
$$

And is rewritten to the state space representation as

$$
\left\{\begin{array}{c}
\dot{\boldsymbol{x}}(t)=\left[\begin{array}{cc}
-10.78 & 0 \\
1 & 0
\end{array}\right] \boldsymbol{x}(t)+\left[\begin{array}{l}
1 \\
0
\end{array}\right] u(t-0.04) \\
y(t)=\left[\begin{array}{ll}
0 & 339.6
\end{array}\right] \boldsymbol{x}(t)
\end{array}\right.
$$

We choose the parameters optionally as

$$
\begin{gathered}
\boldsymbol{Q}_{e}=\left[\begin{array}{ccc}
90 & 0 & 0 \\
0 & 90 & 0 \\
0 & 0 & 90
\end{array}\right], r_{e}=1, \\
\boldsymbol{Q}_{s}=\left[\begin{array}{cc}
100 & 0 \\
0 & 3
\end{array}\right], r_{s}=1 \quad, \varepsilon=0.5,
\end{gathered}
$$

$$
\boldsymbol{Q}_{o}=\left[\begin{array}{ll}
1 & 0 \\
0 & 1
\end{array}\right], r_{o}=1,
$$

$K=10$, and $\eta=70$.

From (7.2), the feedback gain $\boldsymbol{F}_{\boldsymbol{1}}$ and servo gain $F_{2}$ can be calculated by

$$
\begin{aligned}
\boldsymbol{F} & =\left[\begin{array}{ll}
\boldsymbol{F}_{1} & F_{2}
\end{array}\right] \\
& =\left[\begin{array}{lll}
21.2275 & 454.1353 & 9.4868
\end{array}\right],
\end{aligned}
$$

And the switching matrix $S$ is calculated by

$$
\boldsymbol{S}=\left[\begin{array}{ll}
5.0754 & 15.0547
\end{array}\right]
$$

Finally, the predictor gain $\boldsymbol{L}_{o}$ is

$$
\boldsymbol{L}_{o}=\left[\begin{array}{ll}
87.3142 & 1.2305
\end{array}\right]^{T}
$$

Fig. 3 shows the simulation results of the sliding mode control with a state predictor for the time delay control system. In Fig. 3, a unit step is introduced at time $t=0.0$ [sec] as $r(t)$. An input side disturbance $D(t)=-0.1$ is introduced at time $t=3.0[\mathrm{sec}]$.

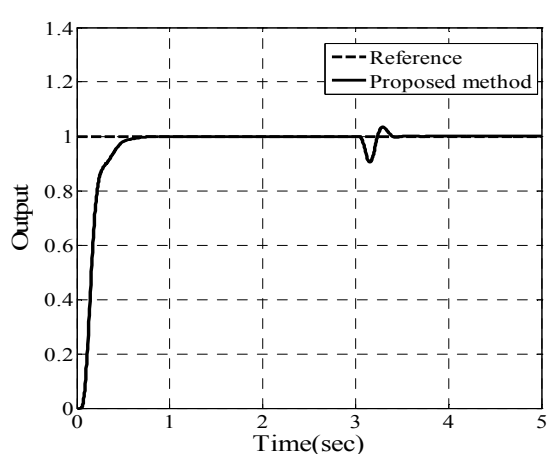

Fig. 3. Simulation results for the proposed method.

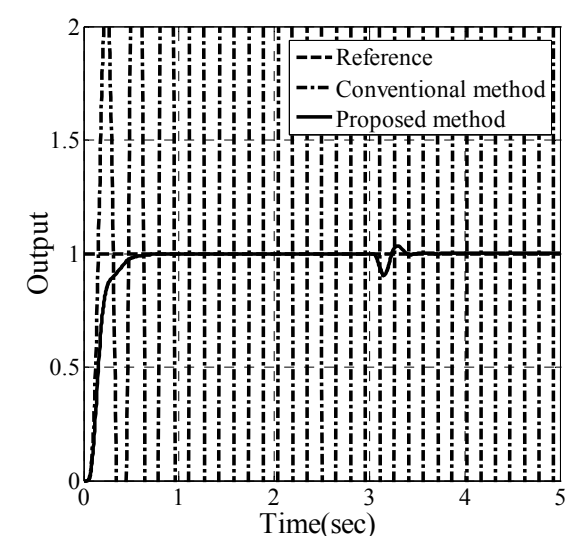

Fig. 4. Comparison between the proposed method and conventional method.

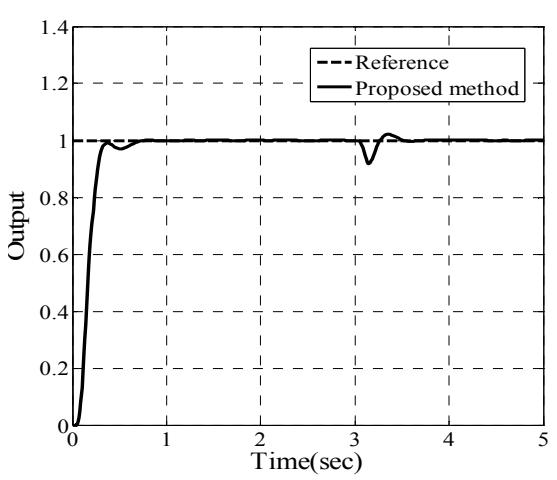

Fig. 5. Robustness of the proposed method. 
Next, we compare the proposed method with conventional method. The conventional method consists of the plant, observer, and servo system, but the predictor is not included. Fig. 4 shows the comparison of simulation results of the proposed method with conventional method.

Finally, we have confirmed the robustness of the proposed method. SMC is known as being robust, but the added state predictor is unknown. We rewrite $G(s)$ as $G_{e}(s)$, and $G_{e}(s)$ is defined as

$$
\begin{aligned}
& G_{e}(s)=\frac{339.6}{s\left(s+10.78 \times e_{m}\right)} e^{-0.04}, \\
& e_{m}=1.5,
\end{aligned}
$$

where $e_{m} \in \mathbb{R}^{1 \times 1}$ is the modeling error. The state predictor parameters $(\boldsymbol{A}, \boldsymbol{B}, \boldsymbol{C}, L)$ are the same as (7.2). Fig.5 shows the robustness of the proposed method.

\section{CONCLUSION}

In this study, we have controlled a plant with time delay using SMC with a state predictor. Simulation results show that the proposed method is improved when compared with the conventional method, and we have confirmed that the proposed method is effective for an input side disturbance.

Furthermore, we have confirmed the robustness, which is one of the characteristics of SMC when we add a state predictor.

\section{REFERENCES}

[1] M. C. Saaj, B. Bandyopadhyay, and H. Unbehauen, "A New Algorithm for Discrete-Time Sliding-Mode Control Using Fast Output Sampling Feedback," IEEE Transaction On Industrial Electronics, vol. 49, no. 3 , pp. 518-523, 2002.

[2] M. Uchida, T. Yabumi, Y. Morita, and H. Kando, "Positioning Control of Linear Motor via Sliding Mode Control System with Variable Switching Hyperplane," IEEE J Trans. EIS, vol. 126, no. 9, pp. 1112-1118, 2006.

[3] S. Lee and C. C. Chung, "Sliding Mode Control Design Using Fast Output Sampling," in Proceeding of the 42nd IEEE, Conference on Decision and Control, pp. 3543-3548, Maui, Hawaii USA, December 2003.

[4] Y. Kanai, M. Kasahara, and Y. Mori. "Discrete-Time Sliding Mode Control with Time Varying Switching Hyperplane which is Moving in the Specified Region," IEEE J Trans. EIS, vol. 130, no. 10, pp.1833-1839, 2010.

[5] H. Kanoh, N. Ehara, H. Kobayashi, and O. Ono, "Analysis and Control of Dynamical Systems," Corona Publishing Co., Ltd, Tokyo, 1991.

[6] M. Miyazaki, System controls I, Ohmsha, 2003.

[7] M. Miyazaki, System control II, Ohmsha, 2008.

[8] F. Golnaraghi and B. C. Kuo, Automatic Control Systems, Wiley \& Sons, Inc, 2010.

[9] Y. Chen and T. Mita, "Adaptive Robust Sliding Mode Control," T. IEE, Japan, vol.113, pp. 203-210, 1993.

[10] S. Sangwongwanich, G. Yonemoto, T. Huruhashi, and S. Ohkuma, "Design of Sliding Observer for Estimation of Rotor Flux of Induction Motors," T.IEE, Japan, vol.110, no. 4, pp. 279-288, 1990.

[11] T. L. Chern and Y. C. Wu, "Design of integral variable structure controller and application to electrohydraulic velocity servo systems," IEEE Proceedings- $D$, vol. 138, no. 5, pp. 439-444, 1991.

[12] T. L. Chern and Y. C. Wu, "An Optimal Variable Structure Control with Integral Compensation for Electrohydraulic Position Servo Control Systems," IEEE Transactions on Industrial Electronics, vol. 39, no. 5, pp. 460-463, 1992.

[13] N. Hamada, N. Matsumoto, and T. Takahashi, "Introduction to Modern Control Theory," Corona Publishing Co., Ltd, Tokyo, 2011.
[14] K. Nonami and T. Hongqi, "Sliding Mode Control," Corona Publishing Co., Ltd, Tokyo, 2010, (in Japanese).

[15] Y. Nishimura, J. Kimura, and Y. Yonezawa, "On a Construction of a State Predictor for a System with Time Delay," SICE, vol. 28, no. 11, pp. 1377-1379, 1992.

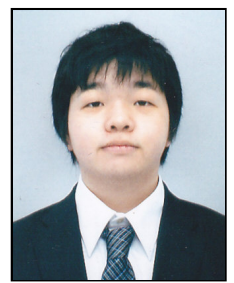

T. Ishibashi was born in Aug. 1990 in Hokkaido, Japan. He is currently studying for the B. E. degree at Undergraduate School of Science and Technology, Meiji University. His research interests with sliding mode control (SMC), adaptive observer and state predictor for a plant with time delay. He is a student member of the IEEJ of Japan.

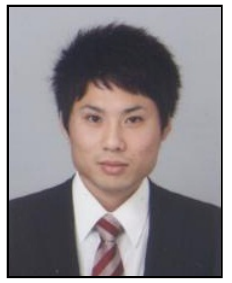

K. Kawaguchi was born in Feb. 1990 in Wakayama, Japan. He received the B. E. degree in Electronics and Bioinformatics from Meiji University, Kawasaki, Japan, in 2011. He is currently studying toward the M. E. degree at Graduate School of Science and Technology, Meiji University. His research interests include Dahlin Algorithm for a plant with time delay and its application to pneumatic equipment.

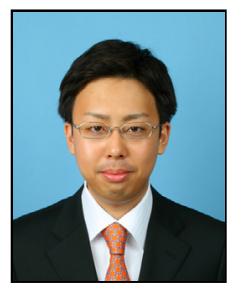

H. Shibasaki was born in Nov. 1988 in Tokyo, JAPAN. He received the B. E. degree in Electronics and Bioinformatics from Meiji University, Kawasaki, Japan, in 2011 . He is currently studying toward the M E. degree at Graduate School of Science and Technology, Meiji University. His research interests include continuous and digital control for a plant with integrator, and an unstable plant and its application to pneumatic equipment. He is a student member of the IEEE, and the IEEJ of Japan.

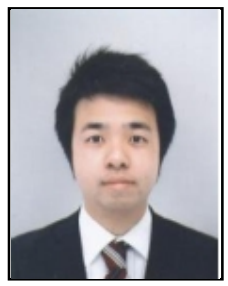

R. Tanaka was born in Oct. 1987 in Fukuoka, Japan. $\mathrm{He}$ received the B. E. degree in Electronics and Communications from Meiji University, Kawasaki, Japan, in 2010. He received the M. E. degree in Graduate School of Science and Technology, Meiji University, in 2012. He is currently studying toward the Dr. Eng. degree at Graduate School of Science and Technology, Meiji University. His research interests include Speech Signal Processing, Noise Reduction, and digital Control Systems.

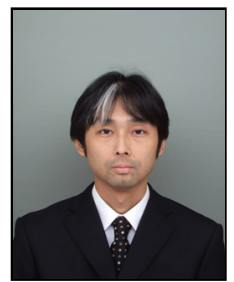

T. Murakami received the B.E., M.E., and Dr.Eng. degree in Electrical Engineering from Meiji University, Japan, in 2000, 2002, and 2005, respectively. From 2005 to 2006, he worked as a Research Assistant at the Department of Electrical Engineering, Meiji University. From 2006 to 2009, he was a Research Fellow of Japan Society for the Promotion of Science (JSPS). From 2009 to 2012, he worked as an Assistant Professor at the Department of Monozukuri Engineering, Tokyo Metropolitan College of Industrial Technology. In 2012, he joined the Department of Electronics and Bioinformatics, Meiji University, where he is currently a Lecturer. His research interests include speech signal processing, blind signal processing, and digital signal processing. He is a member of the IEICE.

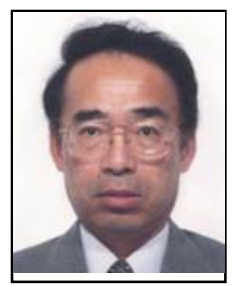

Y. Ishida was born in Feb. 1947 in Tokyo, Japan. He received the B. E., M. E., and Dr. Eng. Degrees in Electrical Engineering, Meiji University, Kawasaki, Japan, in 1970, 1972, and 1978, respectively. In 1975 he joined the Department of Electrical Engineering, Meiji University, as a research Assistant and became a Lecturer and an Associate Professor in 1978 and 1981, respectively. He is currently a Professor at the Department of Electronics and Bioinformatics, Meiji University. His current research interests include signal processing, speech analysis and recognition, and digital control. $\mathrm{He}$ is a member of the IEEE, and the IEICE of Japan. 